\title{
ON THE SUPPOSED GRAVITATIONAL ATTRACTION BETWEEN TWO REVOLVING ELECTRONS.
}

By G. A. Schotт.

I. In a recent paper ${ }^{1} \mathrm{~A}$. C. Crehore claims to have proved that two electrons, revolving with uniform velocities in circular orbits about positive centers, in addition to their electrostatic repulsion exert on each other a residual attraction, which varies inversely as the square of the distance between the two centers. For diamond he finds this residual attraction to be immensely greater than the gravitational attraction and hence concludes, quite legitimately if his result be correct, that the fundamental equations of the accepted Electron Theory require substantial modification. It is obviously imperative that Crehore's result be either verified or disproved.

The residual attraction is obtained from the electric force exerted on an electron (I) by an electron (2), the magnetic effect being omitted because it is smaller than the electric effect for low speed electrons. This is true when the comparison is made with the large electrostatic repulsion, but it cannot be assumed without question when the comparison is made with the small residual attraction. Thus it is desirable to take the magnetic effect into account $a b$ initio, although it will be found to be inappreciable on the average for an amorphous medium, but not necessarily so for a crystal; nor is the investigation rendered more complicated by doing so. A great simplification however is effected by omitting at the outset all terms which are of higher degree than the second in the inverse distance between the two centers, for such terms obviously cannot contribute to gravitational attraction. The following investigation is based on Crehore's equations for the electric part of the mechanical force (loc. cit., pp. 453, 454), which have been verified, except some obvious misprints, e. g., $a_{2}$ for $a_{1}$ in the last term of (49).

2. The Mechanical Force between Trwo Moving Electrons. - The complete expression for the mechanical force is given by Crehore (equation (15), p. 448) in the usual form. We have also $R \mathbf{H}=\mathbf{R} \times \mathbf{E}$, so that $R\left(\mathbf{q}_{1} \times \mathbf{H}\right)=\mathbf{q}_{1} \times(\mathbf{R} \times \mathbf{E})=\mathbf{R}\left(\mathbf{q}_{1} \cdot \mathbf{E}\right)-\mathbf{E}\left(\mathbf{q}_{1} \cdot \mathbf{R}\right)$. Hence the total mechanical force $\mathbf{F}$ is found from the equation

$$
\mathbf{F} / e_{1}=\mathbf{E}\left\{\mathbf{I}-\left(\mathbf{q}_{1} \cdot \mathbf{R}\right) / c R\right\}+\mathbf{R}\left(\mathbf{q}_{1} \cdot \mathbf{E}\right) / c R .
$$

${ }^{1}$ A. C. Crehore, Phys. Rev., Sec. Ser., Vol. IX., p. 445, June, I9r 7. 
Its component along the line of centers is given by

$$
(\mathbf{F} \cdot \mathbf{r}) / r e_{1}=(\mathbf{E} \cdot \mathbf{r})\left\{\mathbf{I}-\left(\mathbf{q}_{1} \cdot \mathbf{R}\right) / c R\right\} / r+(\mathbf{R} \cdot \mathbf{r})\left(\mathbf{q}_{1} \cdot \mathbf{E}\right) / R r c .
$$

The components of the electric force $\mathbf{E}$ in the directions $i, j, k$ fixed in the orbit of the first electron are given by Crehore's equations (48), (49) and (5o), pp. 453 and 454. As we have already stated above we shall only retain terms of the orders $r^{-1}$ and $r^{-2}$; in the latter we may replace $R$ by $r$, but we must keep $R$ in the former as well as in the circular functions $C_{2}$ and $S_{2}$. It will be convenient to replace the coördinates $(x, y, z)$ by their values $r(X, Y, Z)$ where $X, Y, Z$ are the direction cosines of the line of centers referred to the axes of $i, j, k$ fixed in the first orbit; we shall also introduce its direction cosines $\xi, Y, \zeta$ relative to the axes of $i^{\prime}, j^{\prime}, k^{\prime}$ fixed in the second orbit. Then we have

$$
\xi=X \cos \alpha+Z \sin \alpha, \quad \zeta=Z \cos \alpha-X \sin \alpha .
$$

Rearranging the terms retained in Crehore's equations more conveniently for our purpose we find

$$
\begin{aligned}
(\mathbf{E} \cdot \mathbf{i}) & r^{2} A^{3} / e_{2} \\
= & -X\left\{\mathrm{I}-\left(C_{1} C_{2}+S_{1} S_{2} \cos \alpha\right) \beta_{2}{ }^{2} a_{1} / a_{2}+\left(\xi S_{2}+Y C_{2}\right) \beta_{2}{ }^{2} r^{4} / R^{3} a_{2}\right\} \\
& -\cos \alpha\left\{\beta_{2}\left(\mathrm{I}-\beta_{2}{ }^{2}\right) C_{2}-\beta_{2}{ }^{2} S_{2}\left[\xi S_{2}+Y C_{2}-2\left(X S_{1}+Y C_{1}\right) a_{1} / a_{2}\right]\right. \\
& \left.+\beta_{2}{ }^{3} Y r^{3} / R^{2} a_{2}-\beta_{2}{ }^{2} S_{2} r^{4} / R^{3} a_{2}\right\}+S_{1}\left(\xi S_{2}+Y C_{2}\right) \beta_{2}{ }^{2} a_{1} / a_{2}, \\
(\mathbf{E} \cdot \mathbf{j}) r^{2} A^{3} / e_{2} & \\
= & -Y\left\{\mathrm{I}-\left(C_{1} C_{2}+S_{1} S_{2} \cos \alpha\right) \beta_{2}{ }^{2} a_{1 /} a_{2}+\left(\xi S_{2}+Y C_{2}\right) \beta_{2}{ }^{2} r^{4} / R^{3} a_{2}\right\} \\
& +\beta_{2}\left(\mathrm{I}-\beta_{2}{ }^{2}\right) S_{2}+\beta_{2}{ }^{2} C_{2}\left[\xi S_{2}+Y C_{2}-2\left(X S_{1}+Y C_{1}\right) a_{1} / a_{2}\right] \\
& +\beta_{2}{ }^{3} \xi r^{3} / R^{2} a_{2}+\beta_{2}{ }^{2} C_{2} r^{4} / R^{3} a_{2}+C_{1}\left(\xi S_{2}+Y C_{2}\right) \beta_{2}{ }^{2} a_{1} / a_{2}, \\
(\mathbf{E} \cdot \mathbf{k}) & r^{2} A^{3} / e_{2} \\
= & -Z\left\{\mathrm{I}-\left(C_{1} C_{2}+S_{1} S_{2} \cos \alpha\right) \beta_{2}{ }^{2} a_{1} / a_{2}+\left(\xi S_{2}+Y C_{2}\right) \beta_{2}{ }^{2} r^{4} / R^{3} a_{2}\right\} \\
& -\sin \alpha\left\{\beta_{2}\left(\mathrm{I}-\beta_{2}{ }^{2}\right) C_{2}-\beta_{2}{ }^{2} S_{2}\left[\xi S_{2}+Y C_{2}-2\left(X S_{1}+Y C_{1}\right) a_{1} / a_{2}\right]\right. \\
& \left.+\beta_{2}{ }^{3} Y r^{3} / R^{2} a_{2}-\beta_{2}{ }^{2} S_{2} r^{4} / R^{3} a_{2}\right\} .
\end{aligned}
$$

3. In order to find $(\mathbf{E} \cdot \mathbf{r})$, which is $r$ times the component of the electric force along the line of centers, we must multiply (4), (5), (6) by $X, Y, Z$ respectively and add the products together. The large terms of order $r^{-1} a_{2}{ }^{-1}$ in the three equations cancel on account of (3), and we obtain the equation

$$
\begin{aligned}
& (\mathbf{E} \cdot \mathbf{r}) r A^{3} / e_{2}=-\mathbf{I}+\beta_{2}\left(\mathbf{I}-\beta_{2}{ }^{2}\right)\left(Y S_{2}-\xi C_{2}\right)+\beta_{2}{ }^{2}\left(\xi S_{2}+Y C_{2}\right)^{2} \\
& +\left\{C_{1} C_{2}+S_{1} S_{2} \cos \alpha-\left(X S_{1}+Y C_{1}\right)\left(\xi S_{2}+Y C_{2}\right)\right\} \beta_{2}{ }^{2} a_{1} / a_{2} .
\end{aligned}
$$

All large terms have cancelled out; in what remains we may put to a 
first approximation, using Crehore's equations (38), (39) and (43),

$$
A=\mathrm{I}-\left(\mathbf{q}_{2} \cdot \mathbf{R}\right) / c R=\mathrm{I}-\beta_{2}\left(Y S_{2}-\xi C_{2}\right)=K_{2} .
$$

To the same approximation we may put for the factor of $(\mathbf{E} \cdot \mathbf{r}) / r$ in (2), using Crehore's equations (38), (39) and (42),

$$
\mathrm{I}-\left(\mathbf{q}_{1} \cdot \mathbf{R}\right) / c R=\mathrm{I}-\beta_{1}\left(Y S_{1}-X C_{1}\right)=K_{1} .
$$

To find the second term of (2) we multiply (4) by $\beta_{1} C_{1}$, (5) by $-\beta_{1} S_{1}$, in accordance with Crehore's equation (42); and add, obtaining

$$
\left.\begin{array}{rl}
\left(\mathbf{q}_{1} \cdot \mathbf{E}\right) & r^{2} A^{3} / c e_{2} \\
= & \beta_{1}\left(Y S_{1}-X C_{1}\right)\left\{\mathrm{I}-\left(C_{1} C_{2}+S_{1} S_{2} \cos \alpha\right) \beta_{2}{ }^{2} a_{1} / a_{2}\right\} \\
& -\beta_{1} \beta_{2}\left(\mathbf{I}-\beta_{2}{ }^{2}\right)\left(C_{1} C_{2} \cos \alpha+S_{1} S_{2}\right) \\
& +\beta_{1} \beta_{2}{ }^{2}\left(C_{1} S_{2} \cos \alpha-S_{1} C_{2}\right)\left\{\xi S_{2}+Y C_{2}-2\left(X S_{1}+Y C_{1}\right) a_{1} / a_{2}\right\} \\
& +\beta_{1} \beta_{2}{ }_{2}\left\{C_{1} \bar{S}_{2} \cos \alpha-S_{1} \bar{C}_{2}+\left(Y S_{1}-X C_{1}\right)\left(\xi \bar{S}_{2}+Y \bar{C}_{2}\right)\right\} r^{4} / \bar{R}^{3} a_{2} \\
& -\beta_{1} \beta_{2}{ }^{3}\left(Y C_{1} \cos \alpha+\xi S_{1}\right) r^{3} / \bar{R}^{2} a_{2}
\end{array}\right\}
$$

The terms in the three first lines are small and only give rise to terms of order $r^{-2}$ in the force, so that here we may replace $A$ by its approximate value $K_{2}$ as above.

The others, however, bracketed together, are large and give rise to terms of order $r^{-1} a_{2}^{-1}$ in the force, and therefore need special treatment. We must retain terms of order $a_{1} / r$ and $a_{2} / r$ both in $A$ and $R$, and in the functions $S_{2}$ and $C_{2}$ of $R$; these are distinguished for the moment by a bar over them. We find from Crehore's equations (38) and (39)

$$
\left.\begin{array}{rl}
\bar{R}= & r-\left(X S_{1}+Y C_{1}\right) a_{1}+\left(\xi \bar{S}_{2}+Y \bar{C}_{2}\right) a_{2}+\rho / 2 r, \\
\rho= & \left\{\mathrm{I}-\left(X S_{1}+Y C_{1}\right)^{2}\right\} a_{1}^{2}+\left\{\mathrm{I}-\left(\xi S_{2}+Y C_{2}\right)^{2}\right\} a_{2}^{2} \\
& -2\left\{C_{1} C_{2}+S_{1} S_{2} \cos \alpha-\left(X S_{1}+Y C_{1}\right)\left(\xi S_{2}+Y C_{2}\right)\right\} a_{1} a_{2} .
\end{array}\right\} .
$$

The term $\rho / 2 r$ is clearly of the second order, but is required nevertheless as we shall see below. We may write to the first order

whence

$$
\begin{aligned}
R & =r-\left(X S_{1}+Y C_{1}\right) a_{1}+\left(\xi S_{2}+Y C_{2}\right) a_{2}, \\
\left(S_{2}, C_{2}\right) & =(\sin , \cos )\left[\omega_{2}(t-R / c)+\theta_{2}\right],
\end{aligned}
$$

$$
\left(\overline{S_{2}}, \overline{C_{2}}\right)=\left(S_{2}, C_{2}\right)+\left(-C_{2}, S_{2}\right) \beta_{2}(\bar{R}-R) / a_{2} .
$$

Substituting in the first equation (II) and using (8) we obtain

$$
\bar{R}=R+\rho / 2 K_{2} r, \quad\left(\bar{S}_{2}, \bar{C}_{2}\right)=\left(S_{2}, C_{2}\right)+\left(-C_{2}, S_{2}\right) \beta_{2} \rho / 2 K_{2} r a_{2} .
$$


With the same notation we find by Crehore's equation (43) and by (12)

$$
\begin{aligned}
A \bar{R}= & \bar{R}-\left(\mathrm{q}_{2} \cdot \overline{\mathbf{R}}\right) / c \\
= & \bar{K}_{2} r-\left\{X S_{1}+Y C_{1}+\beta_{2}\left(S_{1} \bar{C}_{2} \cos \alpha-C_{1} \bar{S}_{2}\right)\right\} a_{1} \\
& \quad+\left(\overline{\xi S_{2}}+Y \bar{C}_{2}\right) a_{2}+\rho / 2 r,
\end{aligned}
$$

where

$$
\bar{K}_{2}=\mathrm{I}-\beta_{2}\left(Y \bar{S}_{2}-\xi \bar{C}_{2}\right)=K_{2}+\left(\xi S_{2}+Y C_{2}\right) \beta_{2}{ }^{2} \rho / 2 K_{2} r a_{2} .
$$

This is required to the first order for substitution in the last two lines of (Io); bearing in mind that $\rho$ is only of the second order we find to the first order

$$
\begin{aligned}
A \bar{R}=K_{2} r-\left\{X S_{1}+Y C_{1}+\right. & \left.\beta_{2}\left(S_{1} C_{2} \cos \alpha-C_{1} S_{2}\right)\right\} a_{1} \\
& +\left(\xi S_{2}+Y C_{2}\right) a_{2}+\left(\xi S_{2}+Y C_{2}\right) \beta_{2}{ }^{2} \rho / 2 K_{2} a_{2} .
\end{aligned}
$$

From this equation together with (II) we find

$$
\begin{aligned}
r^{4} / A^{3} \bar{R}^{3} a_{2}=r / K_{2}{ }^{3} a_{2}+3\left\{X S_{1}+Y C_{1}+\beta_{2}\left(S_{1} C_{2} \cos \alpha-C_{1} S_{2}\right)\right\} a_{1} / K_{2}{ }^{4} a_{2} \\
\quad-3\left(\xi S_{2}+Y C_{2}\right) / K_{2}{ }^{4}-3\left(\xi S_{2}+Y C_{2}\right) \beta_{2}{ }^{2}\left[\left\{\mathrm{I}-\left(X S_{1}+Y C_{1}\right)^{2}\right\} a_{1}{ }^{2}\right. \\
\quad+\left\{\mathrm{I}-\left(\xi S_{2}+Y C_{2}\right)^{2}\right\} a_{2}{ }^{2}-2\left\{C_{1} C_{2}+S_{1} S_{2} \cos \alpha\right. \\
\left.\left.\quad-\left(X S_{1}+Y C_{1}\right)\left(\xi S_{2}+Y C_{2}\right)\right\} a_{1} a_{2}\right] / 2 K_{2}{ }^{5} a_{2}{ }^{2} \\
r^{3} / A^{3} \bar{R}^{2} a_{2}==r / K_{2}{ }^{3} a_{2}-\left(X S_{1}+Y C_{1}\right) a_{1} / K_{2}{ }^{3} a_{2}+\left(\xi S_{2}+Y C_{2}\right) / K_{2}{ }^{3} \\
\quad+3\left\{X S_{1}+Y C_{1}+\beta_{2}\left(S_{1} C_{2} \cos \alpha-C_{1} S_{2}\right)\right\} a_{1} / K_{2}{ }^{4} a_{2} \\
\quad-3\left(\xi S_{2}+Y C_{2}\right) / K_{2}{ }^{4}-3\left(\xi S_{2}+Y C_{2}\right) \beta_{2}{ }^{2}\left[\left\{\mathrm{I}-\left(X S_{1}+Y C_{1}\right)^{2}\right\} a_{1}{ }^{2}\right. \\
\quad+\left\{\mathrm{I}-\left(\xi S_{2}+Y C_{2}\right)^{2}\right\} a_{2}{ }^{2}-2\left\{C_{1} C_{2}+S_{1} S_{2} \cos \alpha\right. \\
\left.\left.\quad-\left(X S_{1}+Y C_{1}\right)\left(\xi S_{2}+Y C_{2}\right)\right\} a_{1} a_{2}\right] / 2 K_{2}{ }^{5} a_{2} .
\end{aligned}
$$

Lastly we find to the same order by means of (II) and (I2)

$$
\begin{aligned}
C_{1} \bar{S}_{2} & \cos \alpha-S_{1} \bar{C}_{2}+\left(Y S_{1}-X C_{1}\right)\left(\xi \bar{S}_{2}+Y \bar{C}_{2}\right)=C_{1} S_{2} \cos \alpha-S_{1} C_{2} \\
& +\left(Y S_{1}-X C_{1}\right)\left(\xi S_{2}+Y C_{2}\right)-\left\{C_{1} C_{2} \cos \alpha+S_{1} S_{2}\right. \\
& \left.-\left(Y S_{1}-X C_{1}\right)\left(Y S_{2}-\xi C_{2}\right)\right\} \beta_{2}\left[\left\{\mathrm{I}-\left(X S_{1}+Y C_{1}\right)^{2}\right\} a_{1}^{2}\right. \\
& +\left\{\mathrm{I}-\left(\xi S_{2}+Y C_{2}\right)^{2}\right\} a_{2}^{2}-2\left\{C_{1} C_{2}+S_{1} S_{2} \cos \alpha\right. \\
& \left.\left.-\left(X S_{1}+Y C_{1}\right)\left(\xi S_{2}+Y C_{2}\right)\right\} a_{1} a_{2}\right] / 2 K_{2} r a_{2} .
\end{aligned}
$$

Substituting from (7), (9), (Io) and (I2) in (2) and using (8) in the small terms and (I3), (I4) and (I5) in the large ones we obtain the final expression for the mechanical force between the two electrons. From Crehore's equation (38) and our equation (II) we see that $(\mathbf{R} \cdot \mathbf{r})$ and $-R r$ differ only by a quantity of the second order, so that to our approximation the factor of $\left(q_{1} \cdot E\right) / c$ may be put equal to negative unity. In this way we find after some rearrangement 


$$
\left.\begin{array}{c}
(\mathbf{F} \cdot \mathbf{r}) r / e_{1} e_{2}=-K_{1} / K_{2}{ }^{2}+\left(\xi S_{2}+Y C_{2}\right)^{2} \beta_{2}{ }^{2} K_{1} / K_{2}{ }^{3}-\left(Y S_{2}-\xi C_{2}\right) \beta_{2}{ }^{3} K_{1} / K_{2}{ }^{3} \\
+\left\{C_{1} C_{2}+S_{1} S_{2} \cos \alpha\right. \\
\left.-\left(X S_{1}+Y C_{1}\right)\left(\xi S_{2}+Y C_{2}\right)\right\} \beta_{2}{ }^{2} K_{1} a_{1} / K_{2}{ }^{3} a_{2}
\end{array}\right\}
$$

$+\left[\left\{2\left(C_{1} S_{2} \cos \alpha-S_{1} C_{2}\right)\right.\right.$

$$
\left.-\beta_{2}\left(Y C_{1} \cos \alpha+\xi S_{1}\right)\right\}\left(X S_{1}+Y C_{1}\right)
$$

$\left.+\left(C_{1} C_{2}+S_{1} S_{2} \cos \alpha\right)\left(Y S_{1}-X C_{1}\right)\right] \beta_{1} \beta_{2}{ }^{2} a_{1} / K_{2}{ }^{3} a_{2}$

$-\left[3\left\{X S_{1}+Y C_{1}+\beta_{2}\left(S_{1} C_{2} \cos \alpha-C_{1} S_{2}\right\}\left\{C_{1} S_{2} \cos \alpha\right.\right.\right.$

$$
-S_{1} C_{2}-\beta_{2}\left(Y C_{1} \cos \alpha+\xi S_{1}\right)
$$

$\left.+\left(Y S_{1}-X C_{1}\right)\left(\xi S_{2}+Y C_{2}\right)\right\}$

$$
+\beta_{2}\left\{C_{1} C_{2} \cos \alpha+S_{1} S_{2}-\left(Y S_{1}-X C_{1}\right)\left(Y S_{2}-\xi C_{2}\right)\right\} .
$$

$\left\{C_{1} C_{2}+S_{1} S_{2} \cos \alpha\right.$

$$
\left.\left.-\left(X S_{1}+Y C_{1}\right)\left(\xi S_{2}+Y C_{2}\right)\right\}\right] \beta_{1} \beta_{2}^{2} a_{1} / K_{2}{ }^{4} a_{2}
$$

$-3\left\{C_{1} S_{2} \cos \alpha-S_{1} C_{2}+\left(Y S_{1}-X C_{1}\right)\left(\xi S_{2}+Y C_{2}\right)\right.$

$\left\{C_{1} C_{2}+S_{1} S_{2} \cos \alpha\right.$

$$
\left.-\beta_{2}\left(Y C \cos \alpha+\xi S_{1}\right)\right\} \text {. }
$$

$$
\left.\left.-\left(X S_{1}+Y C_{1}\right)\left(\xi S_{2}+Y C_{2}\right)\right\}\left(\xi S_{2}+Y C_{2}\right) \beta_{1} \beta_{2}{ }^{4} a_{1 /} K_{2}{ }^{5} a_{2}\right)
$$

$$
-\left[Y S_{1}-X C_{1}-\beta_{2}\left(\mathrm{I}-\beta_{2}^{2}\right)\left(C_{1} C_{2} \cos \alpha+S_{1} S_{2}\right)\right.
$$$$
+\beta_{2}{ }^{2}\left\{C_{1} S_{2} \cos \alpha-S_{1} C_{2}\right.
$$

$$
\left.\left.-\beta_{2}\left(Y C_{1} \cos \alpha+\xi S_{1}\right)\right\}\left(\xi S_{2}+Y C_{2}\right)\right] \beta_{1} / K_{2}{ }^{3}
$$

$$
+\left(Y S_{1}-X C_{1}\right)\left(\xi S_{2}+Y C_{2}\right)
$$

$$
+3\left[C_{1} S_{2} \cos \alpha-S_{1} C_{2}\right.
$$

$$
\left.-\beta_{2}\left(Y C_{1} \cos \alpha+\xi S_{1}\right)\right]\left(\xi S_{2}+{ }_{2} Y C_{2}\right) \beta_{1} \beta_{2}{ }^{2} / K_{2}{ }^{4}
$$

$$
\begin{gathered}
+\left[\left\{\mathrm{I}-\left(X S_{1}+Y C_{1}\right)^{2}\right\} a_{1}{ }^{2}+\left\{\mathrm{I}-\left(\xi S_{2}+Y C_{2}\right)^{2}\right\} a_{2}{ }^{2}\right]\left\{C_{1} C_{2}\right. \\
\left.\cos \alpha+S_{1} S_{2}-\left(Y S_{1}-X C_{1}\right) \cdot\left(Y S_{2}-\xi C_{2}\right)\right\} \beta_{1} \beta_{2}{ }^{3} / 2 K_{2}{ }^{4} a_{2}{ }^{2} \\
+3\left[C_{1} S_{2} \cos \alpha-S_{1} C_{2}+\left(Y S_{1}-X C_{1}\right)\left(\xi S_{2}+Y C_{2}\right)\right. \\
\left.-\beta_{2}\left(Y C_{1} \cos \alpha+\xi S_{1}\right)\right]\left[\left\{\mathrm{I}-\left(X S_{1}+Y C_{1}\right)^{2}\right\} a_{1}{ }^{2}\right. \\
\left.+\left\{\mathrm{I}-\left(\xi S_{2}+Y C_{2}\right)^{2}\right\} a_{2}{ }^{2}\right]\left(\xi S_{2}+Y C_{2}\right) \beta_{1} \beta_{2}{ }^{4} / 2 K_{2}{ }^{5} a_{2} \\
-\left[C_{1} S_{2} \cos \alpha-S_{1} C_{2}+\left(Y S_{1}-X C_{1}\right)\left(\xi S_{2}+Y C_{2}\right)\right. \\
\left.-\beta_{2}\left(Y C_{1} \cos \alpha+\xi S_{1}\right)\right] \beta_{1} \beta_{2}{ }^{2} r / K_{2}{ }^{3} a_{2} .
\end{gathered}
$$

This equation affords the material for the remainder of the present investigation. It consists of three groups of terms shown bracketed together; the first three lines include the electric force terms used by 
Crehore, together with certain of the magnetic force terms, which are characterized by the presence of the speed $\beta_{1}$ as a factor occurring in the quantity $K_{1}$ defined by (9). The second group of thirteen lines consists of magnetic force terms of the second degree in the circular functions $S_{1}, C_{1}$, which have the frequency $\omega_{1}$, being defined by Crehore's equations (3I); these are accompanied by the circular functions $S_{2}, C_{2}$, of frequency $\omega_{2}$, defined by Crehore's equations (32). The third group of thirteen lines consists of similar functions, but of the first and third degrees in $S_{1}$, $C_{1}$, and in the last two lines of all they include large terms of the order $r / a_{2}$, all the rest being small.

We shall begin by following Crehore's procedure, checking his result and examining his assumptions critically, with a view to explaining his anomalous conclusions. On the basis of this critique we shall then formulate a more correct procedure.

4. Crehore's Process of Time Averaging.-Taking only the terms in the first three lines of (I6) we put $\beta_{1}$ equal to zero and $K_{1}$ equal to unity; this amounts to omitting all magnetic force terms, a procedure adopted by Crehore for the sake of simplicity, but one which requires justification. Moreover Crehore puts the quantity $K_{2}$ also equal to unity, on the ground that the speed $\beta_{2}$ is small, and takes the time averages of $S_{1}, C_{1}, S_{2}, C_{2}$ and of their products equal to zero, and those of their squares equal to one half. On these assumptions we obtain at once

$$
\overline{(\mathbf{F} \cdot \mathbf{r}) r / e_{1} e_{2}}=-\mathrm{I}+\frac{1}{2}\left(\xi^{2}+Y^{2}\right) \beta_{2}{ }^{2}=-\mathrm{I}+\frac{1}{2}\left(\mathrm{I}-\zeta^{2}\right) \beta_{2}{ }^{2} .
$$

The bar over the symbols denotes time average as always hereafter in this paper. The first term, - I, gives the electrostatic force, the second the residual attraction according to Crehore, in full agreement with his equation (54), p. 456, as we see from our equation (3).

5. In examining this procedure closely we notice that the deliberate neglect of the magnetic force terms is of doubtful validity, because, although the speeds $\beta_{1}$ and $\beta_{2}$ are actually small quantities, and therefore the magnetic force terms in (I6) are small compared with some at any rate of the electric force terms in the same equation, they may nevertheless not be small compared with the residual attraction in (I7), which is itself small. This applies more especially to the terms in the last line of (I6), for they have the large factor $r / a_{2}$ and accordingly require particular attention.

A more serious objection may be urged against Crehore's procedure of putting the quantity $K_{2}$ equal to unity on account of the smallness of $\beta_{2}$. In fact small quantities of the order $\beta_{2}{ }^{2}$ are retained in forming the residual attraction, and we shall see below that the terms omitted in 
consequence of this procedure actually suffice to cancel the supposed residual attraction.

Lastly the method of finding time averages is not rigorous. It is indeed allowable to put the time averages of $S_{1}, C_{1}$ equal to zero, for these latter are true circular functions of the time $t$, as we see from Crehore's equation (3I). But the same thing is not true of $S_{2}, C_{2}$, for we see from Crehore's equations (32) and (39), that their argument involves the distance $R$, which is itself a function of $S_{1}, C_{1}, S_{2}, C_{2}$. It is not even a periodic function of the time $t$, unless the angular velocities $\omega_{1}, \omega_{2}$ happen to be commensurable; this Crehore expressly supposes not to be the case, for it is difficult to see how so special an assumption could be of use in explaining so universal a phenomenon as gravitation.

Thus a correct procedure must not only take this fact into account, but must also retain the factors $K_{1}, K_{2}$ in (I6) and include the magnetic force terms in its purview. It may however be stated at once that the third group of terms in (I6), including the large terms in the last line, will disappear from the time average, owing to their being of odd degree in $S_{1}, C_{1}$, and in consequence of the assumed incommensurability of the angular velocities $\omega_{1}, \omega_{2}$.

In order to complete the determination of the average force between the two kinds of electrons we must average the result obtained for all orientations of the two orbits with respect to the line of centers.

6. Correct Procedure of Averaging.-A correct procedure must be based upon Crehore's equations (3I), (32) and our equation (II), which may be written to the first order

$$
\begin{gathered}
\left(S_{1}, C_{1}\right)=(\sin , \cos )\left[\omega_{1} t+\theta_{1}\right], \quad\left(S_{2}, C_{2}\right)=(\sin , \cos )\left[\omega_{2}(t-R / c)+\theta_{2}\right], \\
R=r-\left(X S_{1}+Y C_{1}\right) a_{1}+\left(\xi S_{2}+Y C_{2}\right) a_{2} .
\end{gathered}
$$

When the values of $S_{1}, C_{1}, S_{2}, C_{2}$ are substituted in the last equation, it becomes analogous to the well-known planetary equation of Bessel, a fact which suggests the following convenient substitutions:

$$
\begin{aligned}
\tau & =t-r / c+\left\{\theta_{2}+\tan ^{-1}(Y / \xi)+\pi\right\} / \omega_{2}-\epsilon_{1} \sin \phi / \omega_{1} \\
\phi & =\omega_{1} t+\theta_{1}+\tan ^{-1}(Y / X)+\pi \\
\psi & =\omega_{2}(t-R / c)+\theta_{2}+\tan ^{-1}(Y / \xi)+\pi \\
\delta & =r / c-\left\{\theta_{2}+\tan ^{-1}(Y / \xi)+\pi\right\} / \omega_{2}+\left\{\theta_{1}+\tan ^{-1}(Y / X)+\pi\right\} / \omega_{1} \\
\boldsymbol{\epsilon}_{1} & =\beta_{1} \sqrt{ }\left(X^{2}+Y^{2}\right), \quad \epsilon_{2}=\beta_{2} \sqrt{ }\left(\xi^{2}+Y^{2}\right)
\end{aligned}
$$

where $\beta_{1}=a_{1} \omega_{1} / c, \beta_{2}=a_{2} \omega_{2} / c$ as before. These equations give.

$$
\begin{aligned}
X S_{1}+Y C_{1} & =-\epsilon_{1} \sin \phi / \beta_{1}, & Y S_{1}-X C_{1}=\epsilon_{1} \cos \phi / \beta_{1}, \\
\xi S_{2}+Y C_{2} & =-\epsilon_{2} \sin \psi / \beta_{2}, & Y S_{2}-\xi C_{2}=\epsilon_{2} \cos \psi / \beta_{2} .
\end{aligned}
$$


Substituting from these equations in the expression given above for $R$ we obtain

$$
R=r+\epsilon_{1} \sin \phi \cdot a_{1} / \beta_{1}-\epsilon_{2} \sin \psi \cdot a_{2} / \beta_{2} .
$$

Eliminating $t$ between (I8) and (I9) and using (2I) we obtain

$$
\phi-\epsilon_{1} \sin \phi=\omega_{1}(\tau+\delta) .
$$

From (9), (23) and (26) we find

$$
K_{1}=\mathrm{I}-\epsilon_{1} \cos \phi=\omega_{1} \partial \tau / \partial \phi,
$$

where the differential coefficient is partial, $\epsilon_{1}$ being kept constant. Again, eliminating $t$ and $R$ between (I8), (20) and (25) we obtain

$$
\psi-\epsilon_{2} \sin \psi=\omega_{2} \tau
$$

Lastly we find from (8), (24) and (28)

$$
K_{2}=\mathrm{I}-\epsilon_{2} \cos \psi=\omega_{2} \partial \tau / \partial \psi,
$$

where the differential coefficient is again partial, but with $\epsilon_{2}$ kept constant.

These expressions must be used in (I6), and the result averaged over an interval of time which is very long compared with each of the two periods $2 \pi / \omega_{1}, 2 \pi / \omega_{2}$; this is necessary on account of the incommensurability of the periods. We must multiply by $d t / T$, or more conveniently by $d \tau / T K_{1}$, and integrate with respect to $\tau$ from o to $T$, where $T$ is very large compared with $2 \pi / \omega_{1}$ and $2 \pi / \omega_{2}$. The integrand must be expressed as the sum of a number of products, whose factors are series of sines and cosines of integral multiples of the respective arguments $\omega_{1}(\tau+\delta), \omega_{2} \tau$; this can be effected by means of (23) and (24), combined with (26)-(29), which latter are of the form of the planetary equation of Bessel and lead to series of Bessel Functions. Since $\omega_{1}$ and $\omega_{2}$ are assumed to be incommensurable, the only terms contributing to the time average are products of factors, all of which possess a constant term; all terms involving series without a constant term as factors may be omitted altogether.

For the sake of brevity we shall use the symbols $S$ and $C$ in place of sin and cos respectively.

7. The Electric Force Terms.-For the sake of comparison with Crehore's result we shall commence by considering the electric force terms, which consist of the first group in (16), but we shall retain the factor $K_{1}$, because this facilitates the calculation without making any serious difference to the argument. 
By means of (18)-(24) we obtain

$$
\begin{aligned}
(\mathbf{F} \cdot \mathbf{r}) r / e_{1} e_{2}= & -K_{1} / K_{2}{ }^{2}+S^{2} \psi \cdot \epsilon_{2} K_{1} / K_{2}{ }^{3}-C \psi \cdot \beta_{2}{ }^{2} \epsilon_{2} K_{1} / K_{2}{ }^{3} \\
& +\{Z(X C \phi+Y S \phi) C \psi-\zeta(Y C \phi \\
& -X S \phi) S \psi\} S \alpha \cdot \beta_{1} \beta_{2}{ }^{3} K_{1} a_{1 /} \epsilon_{1} \epsilon_{2} K_{2}{ }^{3} a_{\grave{2}}+\{(C \alpha C \phi C \psi \\
& \left.+S \phi S \psi) \beta_{2}{ }^{2}-S \phi S \psi \cdot \epsilon_{2}{ }^{2}\right\} \beta_{2} \epsilon_{1} K_{1} a_{1} / \beta_{1} \epsilon_{2} K_{2}{ }^{3} a_{2} .
\end{aligned}
$$

When we multiply by $d \tau / T K_{1}$, the factor $K_{1}$ cancels out, producing the simplification alluded to above. We begin with the first line, from which $\phi$ has disappeared entirely with the cancelling out of $K_{1}$; from (28) and (29) we find the well-known Bessel Function expansion

$$
\mathrm{I} / K_{2}=\mathrm{I} /\left(\mathrm{I}-\epsilon_{2} C \psi\right)=\mathrm{I}+2 \sum_{1}^{\infty} J_{k}\left(k \epsilon_{2}\right) \cos k \omega_{2} \tau .
$$

Multiply this equation by $\epsilon_{2}$ and differentiate the product partially with respect to $\epsilon_{2}$, keeping $\tau$ constant and treating $\psi$ as a function of $\epsilon_{2}$ as well as of $\tau$. Then $\partial \psi / \partial \epsilon_{2}=\sin \psi / K_{2}$, by (28), and we find

$$
\mathrm{I} / K_{2}{ }^{2}-S^{2} \psi \cdot \epsilon_{2}{ }^{2} / K_{2}{ }^{3}=\mathrm{I}+2 \sum_{1}^{\infty}\left\{J_{k}\left(k \epsilon_{2}\right)+k \epsilon_{2} J_{k}{ }^{\prime}\left(k \epsilon_{2}\right)\right\} \cos k \omega_{2} \tau .
$$

We may also write

$$
C \psi / K_{2}{ }^{3}=\epsilon_{2} /\left(\mathbf{I}-\epsilon_{2}^{2}\right)^{3 / 2}+\sum_{1}^{\infty} \dot{A_{k}} \cos k \omega_{2} \tau
$$

The constant is easily verified by means of (29), whilst the values of the coefficients $A_{k}$ are not required for our purpose. When we substitute from (32) and (33) in the first line of (30) and average with respect to the time, all terms disappear except the constants, and we find

$$
(\mathbf{F} \cdot \mathbf{r}) r / e_{1} e_{2}=-\mathrm{I}-\beta_{2}{ }^{2} \epsilon_{2}{ }^{2} /\left(\mathrm{I}-\epsilon_{2}{ }^{2}\right)^{3 / 2} .
$$

This expression gives the complete value of the right-hand member of (30) when averaged, for the last three lines in every term involve either $S \phi$ or $C \phi$ as a factor. But we find in the usual way from (26)

$$
\begin{gathered}
S \phi=2 \sum_{1}^{\infty}\left(k \epsilon_{1}\right)^{-1} J_{k}\left(k \epsilon_{1}\right) \sin k \omega_{1}(\tau+\delta), \\
C \phi=2 \sum_{1}^{\infty} k^{-1} J_{k}\left(k \epsilon_{1}\right) \cos k \omega_{1}(\tau+\delta) .
\end{gathered}
$$

These series have no constant term and therefore contribute nothing to the time average, so that (34) really gives the complete expression required. 
Comparing (34) with ( $\mathrm{I} 7$ ) we see that Crehore's residual attraction has completely disappeared and been replaced by an additional small repulsion. This result is true to every order of $\beta_{1}$ and $\beta_{2}$, and not merely approximately. On examining the process in detail we see that the inverse powers of $K_{2}$, which occur in (32) and (33), on expansion give terms involving $\epsilon_{2}{ }^{2} C^{2} \psi$, and these on averaging not only annul Crehore's residual attraction, but give in addition the lowest terms in the repulsion.

8. The Magnetic Force Terms.-We must now complete our result by taking into account the magnetic force terms consisting of the last two groups in (I6). They differ from the first group by the absence of the factor $K_{1}$ in the numerators, and the consequent appearance in the process of averaging of this factor in all the denominators, in addition to the powers of $K_{2}$ already present. We may dispose of the third group of terms at once, for, as we have seen above, they are of odd degree in $S \phi$ and $C \phi$, the highest power being the third. It follows that each term of the third group involves one or other of the four factors $\left(S \phi, C \phi, S_{3} \phi, C_{3} \phi\right) / K_{1}$. But we easily deduce the following expansions from (26) and (27)

$$
\begin{aligned}
\operatorname{Si\phi } / K_{1} & =\operatorname{Si\phi } /\left(\mathbf{I}-\epsilon_{1} C \phi\right) \\
& =\sum_{1}^{\infty}\left\{J_{k-i}\left(k \epsilon_{1}\right)-J_{k+i}\left(k \epsilon_{1}\right)\right\} \sin k \omega_{1}(\tau+\delta), \\
C i \phi / K_{1} & =C i \phi /\left(\mathbf{I}-\epsilon_{1} C \phi\right) \\
& =\sum_{1}^{\infty}\left\{J_{k-i}\left(k \epsilon_{1}\right)+J_{k+i}\left(k \epsilon_{1}\right)\right\} \cos k \omega_{1}(\tau+\delta) .
\end{aligned}
$$

Neither series has a constant term and therefore contributes nothing to the time average. Thus all the terms of the third group disappear on averaging, confirming what was said above.

Turning now to the second group of terms in (I6), which are all of the second degree in $S \phi, C \phi$, we see that each term involves one or other of the three factors (I, S2 $\left.\phi, C_{2} \phi\right) / K_{1}$, of which the last two vanish on averaging on account of (37) and (38). The first factor only arises from the two square factors $\left(S^{2} \phi, C^{2} \phi\right) / K_{1}$, so that all terms of the second group may be neglected except those which contain these two factors. From (26) and (27) we obtain the expansion

$$
\mathrm{I} / K_{1}=\mathrm{I} /\left(\mathrm{I}-\epsilon_{1} C \dot{\phi}\right)=\mathrm{I}+2 \sum_{1}^{\infty} J_{k}\left(k \epsilon_{1}\right) \cos k \omega_{1}(\tau+\delta)
$$

analogous to (3I). Hence we see that the two square factors $\left(S^{2} \phi\right.$, $\left.C^{2} \phi\right) / K_{1}$ may be replaced by $\mathrm{I} / 2$, and the remaining quadratic factor $S \phi C \phi / K_{1}$ by o, wherever these factors occur in (I6). 
Again, an examination of the second group of (I6) shows that each term involves one of the factors (I, $S \psi, C \psi) / K_{2}{ }^{3},\left(\mathrm{I}, S \psi, C \psi, S^{2} \psi, S \psi C \psi\right.$, $\left.C^{2} \psi\right) / K_{2}{ }^{4}$ and $\left(S^{2} \psi, S \psi C \psi, C^{2} \psi, S^{3} \psi, S^{2} \psi C \psi, S \psi C^{2} \psi, C^{3} \psi\right) / K_{2}{ }^{5}$. The factors, which involve odd powers of $S \psi$, being odd functions of $\psi$, can be expanded in series of sines of multiples of $\omega_{2} \tau$ without a constant term and may therefore be replaced by $o$. The remaining factors are even functions of $\psi$ and can be expanded in series of cosines of multiples of $\omega_{2} \tau$. Their constant term must be determined, but their other coefficients are not required for our purpose. In order to find the constant term of any factor we must multiply it by $\omega_{2} d \tau / 2 \pi$ and integrate over the period $2 \pi / \omega_{2}$, or, what amounts to the same thing and is more convenient, we may multiply it by $K_{2} d \psi / \pi$ and integrate from o to $\pi$. Owing to the presence of powers of $K_{2}$ in the denominators it is best to change the variable from $\psi$ to another quantity $\chi$ which is defined by the transformation

$$
\begin{array}{rlrl}
K_{2}=\mathrm{I}-\epsilon_{2} C \psi & =\left(\mathrm{I}-\epsilon_{2}{ }^{2}\right) /\left(\mathrm{I}+\epsilon_{2} C \chi\right), & d \psi / K_{2}=d \chi / \sqrt{ }\left(\mathrm{I}-\epsilon_{2}{ }^{2}\right), \\
C \psi / K_{2} & =\left(\epsilon_{2}+C \chi\right) /\left(\mathrm{I}-\epsilon_{2}{ }^{2}\right), & & S \psi / K_{2}=S \chi / \sqrt{ }\left(\mathrm{I}-\epsilon_{2}{ }^{2}\right) .
\end{array}
$$

On evaluating the integrals thus obtained we easily find the following values for the constant terms of the factors indicated:

$$
\begin{aligned}
& \left.\begin{array}{l}
\mathrm{I}, C \psi) / K_{2}{ }^{3}=\left(\mathrm{I}, \epsilon_{2}\right) /\left(\mathrm{I}-\epsilon_{2}{ }^{2}\right)^{3 / 2}, \\
\left(\mathrm{I}, C \psi, S^{2} \psi, C^{2} \psi\right) / K_{2}{ }^{4}=\left(2+\epsilon_{2}{ }^{2}, 3 \epsilon_{2}, \mathrm{I}-\epsilon_{2}{ }^{2}, \mathrm{I}+2 \epsilon_{2}{ }^{2}\right) / 2\left(\mathrm{I}-\epsilon_{2}{ }^{2}\right)^{5 / 2}, \\
\left(S^{2} \psi, C^{2} \psi, S^{2} \psi C \psi, C^{3} \psi\right) / K_{2}{ }^{5} \\
\quad=\left(\mathrm{I}-\epsilon_{2}{ }^{2}, \mathrm{I}+4 \epsilon_{2}{ }^{2}, \epsilon_{2}-\epsilon_{2}{ }^{3}, 3 \epsilon_{2}+2 \epsilon_{2}{ }^{3}\right) / 2\left(\mathrm{I}-\epsilon_{2}{ }^{2}\right)^{7 / 2} .
\end{array}\right\}
\end{aligned}
$$

We may sum up the results of this section briefly as follows:

(I) Any term of (I6), which is of odd degree in $S \phi, C \phi$, separately, whatever factors involving $\psi$ it may have, vanishes on averaging and may be omitted. Thus we may omit the whole of the third group.

(2) The square terms $S^{2} \phi, C^{2} \phi$ may be replaced by I/2.

(3) All factors of odd degree in $S \psi$ vanish on averaging, and corresponding terms of the second group of (I6) may be omitted.

(4) All other factors involving $\psi$, whatever their degree in $C \psi$, remain and may be replaced by their constant terms given by (40).

9. In order to shorten the formulæ as much as possible we shall find it convenient to express the seven quantities $X, Y, Z, \xi, \zeta, \cos \alpha, \sin \alpha$ in terms of three of them by means of equations (3) and the usual relations between three direction cosines, choosing for this purpose $Z, \zeta$ and $\cos \alpha$. Nevertheless we shall use the symbols $X, Y$, $\xi$, wherever it conduces to 
brevity to do so. We write

$$
\begin{aligned}
\mu=\cos \alpha, \nu=\sin \alpha=\sqrt{ }\left(\mathbf{I}-\mu^{2}\right), \Sigma & =\sqrt{ }\left(X^{2}+Y^{2}\right)=\sqrt{ }\left(\mathbf{I}-Z^{2}\right), \\
\sigma & =\sqrt{ }\left(\xi^{2}+Y^{2}\right)=\sqrt{ }\left(\mathbf{I}-\zeta^{2}\right) .
\end{aligned}
$$

Hence we obtain by means of (3)

$$
\nu X=Z \mu-\zeta, \quad \nu \xi=Z-\zeta \mu, \quad \nu Y=\sqrt{ }\left(\nu^{2}-Z^{2}-\zeta^{2}+2 Z \zeta \mu\right)
$$

Also we find using (4I) in (22), (23) and (24)

$$
\left.\begin{array}{c}
\epsilon_{1}=\beta_{1} \sqrt{ }\left(\mathrm{I}-Z^{2}\right)=\beta_{1} \Sigma, \epsilon_{2}=\beta_{2} \sqrt{ }\left(\mathrm{I}-\zeta^{2}\right)=\beta_{2} \sigma \\
X S_{1}+Y C_{1}=-\Sigma S \phi, Y S_{1}-X C_{1}=\Sigma C \phi, \\
\xi S_{2}+Y C_{2}=-\sigma S \psi, Y S_{2}-\xi C_{2}=\sigma C \psi, \Sigma S_{1}=Y C \phi-X S \phi, \\
\Sigma C_{1}=-X C \phi-Y S \phi, \sigma S_{2}=Y C \psi-\xi S \psi, \sigma C_{2}=-\xi C \psi-Y S \psi .
\end{array}\right\}
$$

For the purpose of the substitutions to be made in (16) the following expressions will be needed, which can be deduced from (3), (25), (26) and (27) by means of (4I), (42) and (43).

$$
\left.\begin{array}{c}
C_{1} C_{2}+S_{1} S_{2} \cos \alpha=[\{(\mu-Z \zeta) C \psi-Y \zeta \nu S \psi\} C \phi \\
\left.+\left\{Y Z \nu C \psi+\left(\Sigma^{2}+\zeta X \nu\right) S \psi\right\} S \phi\right] / \Sigma \sigma, \\
C_{1} C_{2} \cos \alpha+S_{1} S_{2}=\left[\left\{\left(\Sigma^{2}+\zeta X \nu\right) C \psi-Y Z \nu S \psi\right\} C \phi\right. \\
+\{Y \zeta \nu C \psi+(\mu-Z \zeta) S \psi\} S \phi] / \Sigma \sigma, \\
C_{1} S_{2} \cos \alpha-S_{1} C_{2}=\left[\left\{\left(\Sigma^{2}-Y Z \nu\right) C \psi+\zeta X \nu S \psi\right\} C \phi\right. \\
\left.+\left\{Z X \nu C \psi-\left(\mu \Sigma^{2}-Y \zeta \nu\right) S \psi\right\} S \phi\right] / \Sigma \sigma, \\
S_{1} C_{2} \cos \alpha-C_{1} S_{2}=[-\{Y \zeta \nu C \psi+(\mu-Z \zeta) S \psi\} C \phi \\
\left.+\left\{\left(\Sigma^{2}+\zeta X \nu\right) C \psi-Y Z \nu S \psi\right\} S \phi\right] / \Sigma \sigma, \\
Y C_{1} \cos \alpha+\xi S_{1}=\left[Y Z_{\nu} C \phi-(\mu-Z \zeta) S \phi\right] / \Sigma .
\end{array}\right\}(44)
$$

I0. Taking first the terms of the second group of (I6) which involve $K_{2}^{-3}$ and omitting at once all the terms which vanish owing to the presence of one or more of the quantities $S \phi, C \phi$ or $S \psi$, we find for the others $\left[-2 Z X \nu C \psi S^{2} \phi / \sigma-\beta_{2}(\mu-Z \zeta) S^{2} \phi+(\mu-Z \zeta) C \psi C^{2} \phi / \sigma\right] \beta_{1} \beta_{2}{ }^{2} a_{1} / K_{2}{ }^{3} a_{2}$.

Using rule (2), $\S 8$, the first row of (40), and (43) we obtain for the time average of these terms

$$
-Z X \nu \beta_{1} \beta_{2}{ }^{3} a_{1} / a_{2}\left(\mathrm{I}-\epsilon_{2}^{2}\right)^{3 / 2}
$$

Taking next the terms involving $K_{2}^{-4}$ and omitting vanishing terms as 
before we find for the others

$$
\begin{aligned}
{\left[3 Z X \nu C \psi S^{2} \phi / \sigma\right.} & +3 \beta_{2}(\mu-Z \zeta)\left(S^{2} \phi-S^{2} \psi C^{2} \phi\right) \\
& -3 \beta_{2}{ }^{2}\left\{Y^{2} Z \zeta \nu^{2} C \psi C^{2} \phi+\left(\Sigma^{2}+\zeta X \nu\right)(\mu-Z \zeta) C \psi S^{2} \phi\right\} / \Sigma^{2} \sigma \\
& +3 \beta_{2}\left[\left\{Y \zeta \nu\left(\Sigma^{2}-Y Z \nu\right) C^{2} \psi+(\mu-Z \zeta) \zeta X \nu S^{2} \psi\right\} C^{2} \phi\right. \\
& \left.-\left\{\left(\Sigma^{2}+\zeta X \nu\right) Z X \nu C^{2} \psi+Y Z \nu\left(\mu \Sigma^{2}-Y \zeta \nu\right) S^{2} \psi\right\} S^{2} \phi\right] / \Sigma^{2} \sigma^{2} \\
& -\beta_{2}\left[\left\{\left(\Sigma^{2}+\zeta X \nu\right)(\mu-Z \zeta) C^{2} \psi+Y^{2} Z \zeta \nu^{2} S^{2} \psi\right\} C^{2} \phi\right. \\
& \left.+\left\{Y^{2} Z \zeta \nu^{2} C^{2} \psi+(\mu-Z \zeta)\left(\Sigma^{2}+\zeta X \nu\right) S^{2} \psi\right\} S^{2} \phi\right] / \Sigma^{2} \sigma^{2} \\
& \left.+\beta_{2}(\mu-Z \zeta)\left(S^{2} \psi S^{2} \phi+C^{2} \psi C^{2} \phi\right)\right] \beta_{1} \beta_{2}{ }^{2} a_{1} / K_{2}{ }^{4} a_{2} .
\end{aligned}
$$

Using rule (2), $\S 8$, the second row of (40), and (43) we obtain for the time average of these terms

$$
\begin{gathered}
{\left[{ }_{9} Z X \nu+3(\mu-Z \zeta)\left(\mathrm{I}+2 \epsilon_{2}{ }^{2}\right)-9 \beta_{2}{ }^{2}\left\{Y^{2} Z \zeta \nu^{2}+\left(\Sigma^{2}+\zeta X \nu\right)(\mu-Z \zeta)\right\} / \Sigma^{2}\right.} \\
+3\left[\left\{Y \zeta \nu\left(\Sigma^{2}-Y Z \nu\right)-\left(\Sigma^{2}+\zeta X \nu\right) Z X \nu\right\}\left(\mathbf{I}+2 \epsilon_{2}{ }^{2}\right)\right. \\
\left.+\left\{(\mu-Z \zeta) \zeta X \nu-Y Z \nu\left(\mu \Sigma^{2}-Y \zeta \nu\right)\right\}\left(\mathbf{I}-\epsilon_{2}{ }^{2}\right)\right] / \Sigma^{2} \sigma^{2} \\
-\left\{\left(\Sigma^{2}+\zeta X \nu\right)(\mu-Z \zeta)+Y^{2} Z \zeta \nu^{2}\right\}\left(2+\epsilon_{2}{ }^{2}\right) / \Sigma^{2} \sigma^{2} \\
\left.+(\mu-Z \zeta)\left(2+\epsilon_{2}{ }^{2}\right)\right] \beta_{1} \beta_{2}{ }^{3} a_{1} / 4 a_{2}\left(\mathbf{I}-\epsilon_{2}{ }^{2}\right)^{5 / 2}
\end{gathered}
$$

By means of (4I), (42) and (43) this reduces to

$$
\begin{aligned}
{\left[3 \mu\left({ }_{3} Z^{2}+\mathrm{I}-\epsilon_{2}{ }^{2}\right)\right.} & -7 Z \zeta\left(2+\epsilon_{2}{ }^{2}\right)+3 \beta_{2}{ }^{2}\{(2 \zeta+Z \mu) Y-(\zeta+2 Z \mu) \xi\} \nu \\
& \left.-3 X(Y+\xi) \nu^{2} /\left(\mathrm{I}-\zeta^{2}\right)\right] \beta_{1} \beta_{2}{ }^{3} a_{1} / 4 a_{2}\left(\mathrm{I}-\epsilon_{2}{ }^{2}\right)^{5 / 2} .
\end{aligned}
$$

Lastly, the terms of the second group which involve $K_{2}^{-5}$, when vanishing terms are omitted as before, may be written in the form

$$
\begin{aligned}
& 3\left[\left\{\left(\Sigma^{2}-Y Z \nu\right) C \psi+\left(\zeta X \nu-\Sigma^{2} \sigma^{2}\right) S \psi-\epsilon_{2} Y Z \nu\right\} C \phi\right. \\
& \left.\quad+\left\{Z X \nu C \psi-\left(\mu \Sigma^{2}-Y \zeta \nu\right) S \psi+\epsilon_{2}(\mu-Z \zeta)\right\} S \phi\right] . \\
& {[\{(\mu-Z \zeta) C \psi-Y \zeta \nu S \psi\} C \phi} \\
& \left.\quad+\left\{Y Z \nu C \psi+\left(\Sigma^{2}+\zeta X \nu-\Sigma^{2} \sigma^{2}\right) S \psi\right\} S \phi\right] S \psi \cdot \beta_{1} \beta_{2}{ }^{4} a_{1} / K_{2}{ }^{5} a_{2} \Sigma^{2} \sigma .
\end{aligned}
$$

Omitting vanishing terms, such as those involving $S \phi C \phi$ and those which are of odd degree in $S \psi$, using rule (2), $\S 8$, the third row of (40), and (43) we obtain for the time average

$$
\begin{aligned}
& 3\left[(\mu-Z \zeta)\left(\zeta X \nu-\Sigma^{2} \sigma^{2}\right)-Y \zeta \nu\left(\Sigma^{2}-Y Z \nu\right)+Y^{2} Z \zeta \nu^{2}\right. \\
& \quad-Y Z \nu\left(\mu \Sigma^{2}-Y \zeta \nu\right)+\left(\Sigma^{2}+\zeta X \nu-\Sigma^{2} \sigma^{2}\right) Z X \nu \\
& \left.\quad+\left(\Sigma^{2}+\zeta X \nu-\Sigma^{2} \sigma^{2}\right)(\mu-Z \zeta)\right] \beta_{1} \beta_{2}{ }^{5} a_{1} / 4 \Sigma^{2} a_{2}\left(\mathrm{I}-\epsilon_{2}^{2}\right)^{5 / 2} .
\end{aligned}
$$

By means of (4I), (42) and (43) this reduces to

$$
3\left[3 Z \zeta \sigma^{2}+Z \zeta \nu^{2}+\mu Z^{2} \zeta^{2}-\mu-(\zeta+Z \mu) Y \nu\right] \beta_{1} \beta_{2}{ }^{5} a_{1} / 4 a_{2}\left(\mathrm{I}-\epsilon_{2}{ }^{2}\right)^{5 / 2} .
$$


Collecting the results given in (45), (46) and (47) together and reducing them by means of (42) and (43) we obtain finally for the time average of the magnetic force terms of (I6)

$$
\begin{aligned}
\overline{(\mathbf{F} \cdot \mathbf{r}) r / e_{1} e_{2}}= & \left\{2 Z \zeta+\mu\left(6-Z^{2}\right)\right\} \beta_{1} \beta_{2}{ }^{3} a_{1} / 4 a_{2}\left(\mathrm{I}-\epsilon_{2}{ }^{2}\right)^{3 / 2} \\
& -3\left\{4 Z \zeta+\mu\left(\mathrm{I}-2 Z^{2}\right)-\beta_{2}{ }^{2}(\zeta Y \nu-Z \mu \xi \nu)\right. \\
& \left.+X(Y+\xi) \nu^{2} /\left(\mathrm{I}-\zeta^{2}\right)\right\} \cdot \beta_{1} \beta_{2}{ }^{3} a_{1} / 4 a_{2}\left(\mathrm{I}-\epsilon_{2}{ }^{5}\right)^{5 / 2} .
\end{aligned}
$$

This equation, together with equation (34) for the time average of the electric force terms, gives the complete expression for the time average of the repulsion exerted by the second electron on the first in the direction of the line of centers of their orbits, so far as the inverse square terms are concerned, and that to every order of the two speeds $\beta_{1}$ and $\beta_{2}$. It remains to find its average value for all orientations of the axes of the two orbits.

II. Average Value of the Force for All Orientations.-In order to find this average value we must make some assumption as to the probability of the directions of the axes of the orbits. This depends on the directive force exerted on any one orbit by the electromagnetic fields of all sufficiently near atoms, but without a knowledge of the distribution and orientation of these atoms the directive force cannot be determined. The problem is the same as that which occurs in the determination of the direction of the axis of one magnet of a complex of elementary magnets constituting a natural magnetic body, and a complete solution has not yet been found. Under these circumstances we shall assume that all directions of the axis of the orbit of each electron are equally probable.

The error made on the average with this assumption is in any case unlikely to be serious for any natural body owing to the large number of electrons concerned (crystals?).

We shall take the line of centers as the polar axis from which the direction cosines $Z$ and $\zeta$ are estimated, and shall denote the azimuths of the axes of the two orbits by $\Phi, \Psi$ respectively. Using (4I) and (42) we obtain

$$
\left.\begin{array}{rlrl}
\mu & =Z \zeta+\Sigma \sigma \cos (\Phi-\Psi), & \nu Y & =\Sigma \sigma \sin (\Phi-\Psi), \\
\nu X & =\Sigma\{Z \sigma \cos (\Phi-\Psi)-\zeta \Sigma\}, \quad \nu \xi & =\sigma\{Z \sigma-\zeta \Sigma \cos (\Phi-\Psi)\} .
\end{array}\right\} \text { (49) }
$$

In order to average any expression for all orientations of the axes of the two orbits, in accordance with our assumption of equal probability of all directions, we must multiply it by $d Z d \zeta d \Phi d \Psi / \mathrm{I} 6 \pi^{2}$ and integrate with respect to $\Phi$ and $\Psi$ from 0 to $2 \pi$ and with respect to $Z$ and $\zeta$ from - I to I respectively. 
We shall find it convenient to begin with the magnetic force terms given by (48), for these all disappear on averaging. The terms involving $Y \nu$ obviously do so on account of the presence of the vanishing factor $\sin (\Phi-\Psi)$; those involving $X, \xi$ and $\mu$ reduce to terms which are of odd degree in $Z$ and $\zeta$ separately, on account of the presence of the quantity $\cos (\Phi-\Psi)$. This is multiplied by odd functions of $Z$ and $\zeta$, wherever it occurs squared, and it vanishes on averaging where it occurs in the first degree, as we see from (49). All the terms independent of $\Phi$ and $\Psi$ are of odd degree in $Z$ and $\zeta$ separately, so that after integration with respect to $\Phi$ and $\Psi$ (48) becomes a function of this same type. But a function of this type obviously vanishes when integrated with respect to $Z$ and $\zeta$ between the limits $\pm \mathrm{I}$. Thus the result follows.

Turning now to the electric force terms given by (34), the calculation is much simplified by the fact that the second term on the right, which obviously alone requires any calculation, does not involve $\Phi, \Psi$ or $Z$, but only $\zeta$ implicitly through the quantity $\epsilon_{2}$, determined by (43). In order to evaluate the integral with respect to $\zeta$ we write

$$
\beta_{2} \zeta=\sqrt{ }\left(\mathrm{I}-\beta_{2}{ }^{2}\right) \cdot \sinh u \text {, whence } \sqrt{ }\left(\mathrm{I}-\epsilon_{2}{ }^{2}\right)=\sqrt{ }\left(\mathrm{I}-\beta_{2}{ }^{2}\right) \cdot \cosh u \text {. }
$$

Then we find for the average value of $\epsilon_{2}{ }^{2} /\left(\mathrm{I}-\epsilon_{2}{ }^{2}\right)^{3 / 2}$

$$
\int_{0}^{1} \epsilon_{2}^{2} d \zeta /\left(\mathrm{I}-\epsilon_{2}^{2}\right)^{3 / 2}=\frac{\mathrm{I}}{\mathrm{I}-\beta_{2}^{2}}-\frac{\mathrm{I}}{2 \beta_{2}} \log \frac{\mathrm{I}+\beta_{2}}{\mathrm{I}-\beta_{2}} .
$$

Hence we obtain finally for the average repulsion along the line of centers exerted by the second electron on the first

$$
(\mathbf{F} \cdot \mathbf{r}) / r=-\frac{e_{1} e_{2}}{\gamma^{2}}\left\{\mathrm{I}+\beta_{2}{ }^{2}\left(\frac{\mathrm{I}}{\mathrm{I}-\beta_{2}{ }^{2}}-\frac{\mathrm{I}}{2 \beta_{2}} \log \frac{\mathrm{I}+\beta_{2}}{\mathrm{I}-\beta^{2}}\right)\right\} .
$$

It is to be borne in mind that this expression rests on the assumption that the two periods of revolution are incommensurable. Hence we cannot generally assume that the two speeds are the same, so that we must not put $\beta_{1}$ equal to $\beta_{2}$. Consequently the repulsion exerted by the first electron on the second, which differs from (50) by having $\beta_{1}$ in place of $\beta_{2}$, is different from that exerted by the second on the first, and the Law of Action and Reaction does not hold, except to a first approximation. From a theoretical point of view this may no doubt be urged as an objection to (50) and to the Lorentz-Larmor equations on which it is founded, and used as an argument for modifying them in some such sense as that suggested by Crehore. From an experimental point of view however the deviation is probably not of much moment, for it 
is easily seen by expanding the function of $\beta_{2}$ in $(50)$ that the first power which occurs is the fourth, so that, if $\beta_{2}$ be as large as .or, the relative deviation is less than one hundred-millionth. For this reason I shall not pursue the matter further here.

I2. Summary. -The conclusions of this investigation may be thus summarized:

(I) The residual attraction found by Crehore to exist between two electrons describing circular orbits with uniform speeds, but incommensurable periods, arises from a faulty process of averaging.

(2) When a correct process of averaging is employed, it is replaced by a small residual repulsion, which violates the Law of Action and Reaction, but is not likely ever to be detected by experiment.

(3) The argument based by Crehore on the discrepancy in order of magnitude between his residual attraction and the attraction of gravitation against the correctness of the fundamental equations of the Electron Theory fails, but is replaced by a much weaker argument which may be based on the theoretical violation of the Law of Action and Reaction by the residual repulsion.

University College of Wales, Aberystwyth, England, December 29, I9I7. 